\title{
Aplasia cutis congenita: A case report and literature review
}

\author{
XUNHONG DUAN $^{1}$, GE YANG $^{2}$, DONGQI YU ${ }^{1}$, CHANGLONG YU $^{1}$, BIAO WANG $^{1}$ and YIBIN GUO ${ }^{1}$ \\ Departments of ${ }^{1}$ Burns and Plastic Surgery and ${ }^{2}$ Orthopedic Surgery, The 175 th Hospital of PLA \\ (Affiliated Southeast Hospital of Xiamen University), Zhangzhou, Fujian 363000, P.R. China
}

Received September 23, 2014; Accepted July 9, 2015

DOI: $10.3892 /$ etm.2015.2737

\begin{abstract}
Aplasia cutis congenita (ACC) is a rare condition with an unclear pathogenic mechanism, although the condition has been suggested to occur as a result of the disrupted development or degeneration of skin in utero. ACC associated with fetus papyraceus has been described in numerous studies. Although there have been several reports of ACC, surgical treatment of ACC using the head as a site of donor skin is rarely reported. The present study describes the case of a 1-week-old patient with ACC that was healed by skin grafting, using the scalp as the donor site. The outcome of the procedure showed that the use of the scalp as a donor skin site for grafting is an effective treatment for large and deep ACC lesions arising on sites other than the head.
\end{abstract}

\section{Introduction}

Aplasia cutis congenita (ACC) is a disease that is characterized by a localized or widespread, complete or partial absence or scarcity of skin at birth. The condition is believed to result from the disrupted development or degeneration of skin in utero and was first described by Cordon in 1767 (1). Frieden (2) classified ACC into nine groups according to the pattern and location, underlying causes and anomalies of the condition. Clinically, ACC lesions generally appear as well-demarcated, translucent, ulcerated membranes, through which it is possible to visualize the underlying structures; however the defect may also heal in utero (3). Numerous factors have been considered as possible causes of ACC, including placental infarcts, genetics, teratogenic substances, intrauterine infections and trauma, vascular compromise, amniogenesis, adhesions of the amniotic membrane to the fetal skin, amniotic rupture sequence, ectodermal dysplasia, imperfect neural tube closure and maternal intrapartum drug use (4). Treatment for ACC varies depending on the condition

Correspondence to: Dr Yibin Guo, Department of Burns and Plastic Surgery, The 175th Hospital of PLA (Affiliated Southeast Hospital of Xiamen University), 269 Zhanghua Middle Road, Zhangzhou, Fujian 363000, P.R. China

E-mail: gybsszxwk@163.com

Key words: aplasia cutis congenita, treatment, skin graft, infant of the infant (5), although conservative treatment is the most popular regimen. Although there have been reports of the surgical treatment of ACC, the use of the scalp as a donor site has rarely been reported (6). The present study describes a case of ACC with the lesion on the right lower extremity, which was healed by skin grafting with the head as the donor site.

\section{Case report}

A 1-week-old infant was transferred to the Department of Burns and Plastic Surgery at the 175th Hospital of PLA (Zhangzhou, China) with skin defects on the right hand and lower extremity, which had been present since birth. The 28-year-old mother was healthy, without a history of drug intake, trauma or infectious diseases during pregnancy. The infant was born at term by spontaneous vaginal delivery from non-consanguineous parents. There were no cases of ACC or congenital anomalies of any other organ in the family history of the infant. Weekly ultrasounds of the fetus were not performed during pregnancy. No fetus papyraceus (FP) accompanied the delivery.

The infant presented with irregular linear and large skin defects on the right lower extremity with eschar and small lesions on the right hand (Fig. 1). When the patient first visited the department, the wounds were covered by a simple dressing. Physical examination showed no evidence of other abnormalities. All urine and blood tests, as well as laboratory tests examining kidney and liver function, were normal. Conservative treatment was employed for the first few days following admission to the hospital; however, this did not alleviate the condition. In order to accelerate the healing process and prevent wound infection, skin grafting was performed. The surgical procedure was conducted as follows: i) The patient received a general anesthetic $(3 \mathrm{mg} / \mathrm{kg}$ propofol); ii) razor-thin skin was attained from the right parietal region of the scalp using an electrical dermatome (Zimmer, Inc., Warsaw, IN, USA), and used as a dressing to bind up the donor site; iii) the razor-thin skin should be cleaned of remaining hair prior to grafting; iv) the surface granulation tissue of skin defect was resected, the lesion cleaned was three times, the donor skin was transplanted onto the lesion and the lesion area was binded; and v) operation was completed successfully. The wounds healed within 14 days of admission, with only a little hyperpigmentation (Fig. 2). 


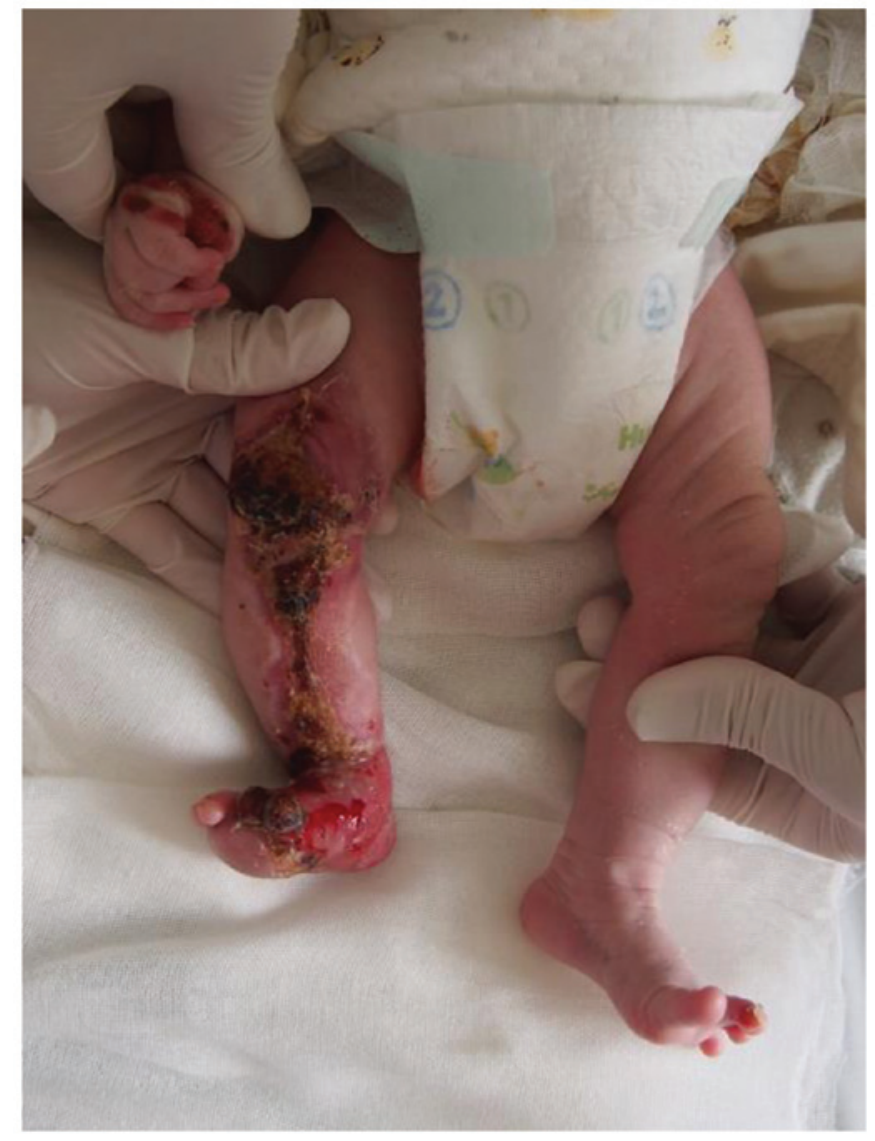

Figure 1. Lesions on the right hand and lower extremity.

\section{Discussion}

ACC is a rare condition with an underlying mechanism that remains to be fully elucidated. To date, only 500 cases have been reported in the literature (5). This congenital skin disorder can occur as an isolated condition, such as the case reported in the present study, or can coexist with other genetic syndromes. One of the factors found to facilitate the development of ACC is maternal cigarette smoking (5). In addition, the association of methimazole, diclofenac sodium, valproic acid, marijuana and cocaine with ACC has already been reported (6).

FP has been found to be closely associated with ACC. The recent increase in the use of in vitro fertilization techniques has caused a marked rise in the incidence of multiple gestation pregnancies (5). FP, or the mortality of the co-twin, which occurs as a result of multi-gestational pregnancies, has been shown to give rise to ACC. Forty-nine cases of FP associated with ACC have been reported in the literature (3). ACC associated with FP (group V of Frieden's classification) (2), is characterized by classic large and symmetrical lesions that are most widely distributed on the trunk and extremities (7). The severity and size of ACC lesions may be determined by the time of mortality of the fetus. When the mortality occurs in the late-first to early-second trimester and prior to 14 weeks' gestation, the lesions tend to be smaller, linear, actuate, triangular and well-demarcated (7).

Several theories have been proposed in order to explain the phenomenon of ACC occurring in the setting of FP $(5,6)$; however, based on the recent evidence, transient hypovolemia

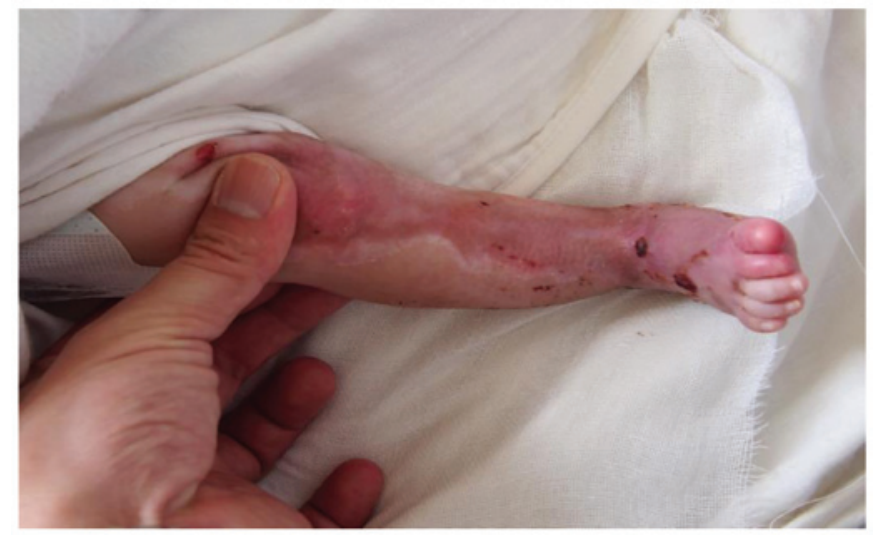

Figure 2. The lesion healed following the skin graft, with little hyperpigmentation.

may be the primary cause (3). Evidence from Doppler ultrasound has demonstrated acute transfusion from the surviving to the dying twin. The ischemia of the skin and other organs of the viable twin may result from hypotension and hypovolemia.

Individuals affected by ACC most commonly exhibit a unique circular defect on the scalp. Skin defects are the primary symptom but can also be accompanied by congenital pulmonary (8) and other malformations, such as lumbosacral sinus tract (3), faun tail nevus and spinal cord malformation $(9,10)$. An ACC scalp defect can be an indicator of internal organ involvement and increase the risk of complications (6). The occurrence of complications can increase the likelihood of a poor outcome. Attention should be paid to the prevention and treatment of complications such as infection, bleeding, electrolyte imbalance from increased epidermal water loss, nutritional deficiency from chronic blood loss and pain at wound dressing (6).

The selection of treatment depends on the condition of the infant and the complications that occur. Multiple treatment regimens have been reported for cases of ACC (6); however, most cases of ACC associated with FP can be healed under conservative treatment, in accordance with the literature $(4,6,11)$. Cerebral damage, bleeding and infections should be carefully monitored when the patient is being conservatively treated with dressing changes. A multidisciplinary approach can be useful. Treatment for ACC is currently controversial and may be conservative, including dressings, surgery or a combination of both. There are a range of conservative dressing options, such as moist dressing, burn cream, dressing with silver sulfadiazine cream and dressings containing epidermal growth factor. Skin grafting and local flaps are the predominant surgical treatment option for ACC. In the literature, a number of cases have been reported in which ACC has been treated using autologous dermis grafts, allogenic dermal grafts and cultured epithelial autografts (12). Skin graft is recommended for large facial defects that are at risk of sagittal sinus thrombosis and cerebral hemorrhage (13). Surgical management should be selected for large and deep lesions that are not healed through conservative treatment.

The present study reported a case of a large lesion on the right lower extremity that bled during dressing changes. In the past, autologous dermal and allogenic dermal grafts, as well as 
cultured epithelial autografts, have been used as treatment for the disease (14). In the present case, an autologous graft was selected. Using the head as the donor site, razor-thin skin was transplanted on the lesion, which healed within a 7-day period without leaving any scars. The lesions are predominantly located on the midline vertex of the scalp, with occasional skull defects, thus the scalp is rarely selected as the donor site. In addition, the fontanelle of the infant is not fully developed, which results in a high risk of cerebral infection. However, in patients without a scalp defect, using the scalp as the donor site may result in fewer additional scars compared with other donor sites. In conclusion, the use of the scalp as a donor site for skin grafting appears to be an effective treatment for large and deep ACC lesions.

\section{References}

1. Cordon M: Extract from a letter describing three children from the same mother born with parts of the extremities devoid of skin. J Med Chir Pharm 26: 556-557, 1767 (In French).

2. Frieden IJ: Aplasia cutis congenita: A clinical review and proposal for classification. J Am Acad Dermatol 14: 646-660, 1986.

3. Mazza JM, Klein JF, Christopher K and Silverberg NB: Aplasia cutis congenita in a setting of fetus papyraceus associated with small fetal abdominal circumference and high alpha-fetoprotein and amniotic acetylcholinesterase. Pediatr Dermatol 32: 138-140, 2013.
4. Blouin MM, Bernard J, Caron F and Auger I: Aplasia cutis congenita of the trunk and scalp associated with fetus papyraceus. Int J Dermatol 50: 733-735, 2011.

5. Ustuner P, Dilek N, Saral Y and Ustüner I: Coexistence of aplasia cutis congenita, faun tail nevus and fetus papyraceus. J Dermatol Case Rep 7: 93-96, 2013.

6. Tempark T and Shwayder TA: Aplasia cutis congenita with fetus papyraceus: Report and review of the literature. Int J Dermatol 51: 1419-1426, 2012.

7. Klein RQ, Robinson DM, Lieber CD and Antaya RJ: Symmetric aplasia cutis congenita associated with fetus papyraceus: Report of two cases. Pediatr Dermatol 28: 467-469, 2011.

8. Schaffer JV, Popiolek DA and Orlow SJ: Symmetric truncal aplasia cutis congenita following multifetal reduction of a sextuplet pregnancy. J Pediatr 153: 860-863, 2008.

9. Louise L, Annabel M, Hubert L, Isabelle G and Gerard L: Fetus papyraceus: Congenital pulmonary anomalies associated with congenital aplasia cutis on the surviving twin. Pediatr Dermatol 30: e143-e145, 2013.

10. Cho AY, Lee SS, Lee Y, Kim CD, Lee JH and Seo YJ: Aplasia cutis congenita with hair collar sign and dermal melanocytosis. Int J Dermatol 51: 745-747, 2012.

11. Kelly BJ, Samolitis NJ, Xie DL and Skidmore RA: Aplasia cutis congenita of the trunk with fetus papyraceus. Pediatr Dermatol 19: 326-329, 2002.

12. Wexler A, Harris M and Lesavoy M: Conservative treatment of cutis aplasia. Plast Reconstr Surg 86: 1066-1071, 1990.

13. Skoufi G, Lialios G, Plachouras N, Kutsogiannis D and Mperis A: Aplasia cutis congenita: Successful conservative treatment. Pediatr Int 48: 507-509, 2006.

14. Anderl H, Frisch H and Hussl H: Successful treatment of an extensive form of aplasia cutis congenita by dermis grafts. Chir Plast (Berl) 5: 183-190, 1980. 